\title{
Trace inequalities of Cassels and Grüss type for operators in Hilbert spaces
}

\author{
Sever S. Dragomir \\ Mathematics, School of Engineering \& Science \\ Victoria University, Australia \\ DST-NRF Centre of Excellence in the Mathematical and \\ Statistical Sciences, \\ School of Computer Science and Applied Mathematics, \\ University of the Witwatersrand, South Africa \\ email: sever.dragomir@vu.edu.au
}

\begin{abstract}
Some trace inequalities of Cassels type for operators in Hilbert spaces are provided. Applications in connection to Grüss inequality and for convex functions of selfadjoint operators are also given.
\end{abstract}

\section{Introduction}

Let $\overline{\mathbf{a}}=\left(a_{1}, \ldots, a_{n}\right)$ and $\overline{\mathbf{b}}=\left(b_{1}, \ldots, b_{n}\right)$ be two positive $n$-tuples with

$$
0<m_{1} \leq a_{i} \leq M_{1}<\infty \text { and } 0<m_{2} \leq b_{i} \leq M_{2}<\infty ;
$$

for each $i \in\{1, \ldots, n\}$, and some constants $m_{1}, m_{2}, M_{1}, M_{2}$.

The following reverses of the Cauchy-Bunyakovsky-Schwarz inequality for positive sequences of real numbers are well known:

a) Pólya-Szegö's inequality [44]:

$$
\frac{\sum_{k=1}^{n} a_{k}^{2} \sum_{k=1}^{n} b_{k}^{2}}{\left(\sum_{k=1}^{n} a_{k} b_{k}\right)^{2}} \leq \frac{1}{4}\left(\sqrt{\frac{M_{1} M_{2}}{m_{1} m_{2}}}+\sqrt{\frac{m_{1} m_{2}}{M_{1} M_{2}}}\right)^{2} .
$$

2010 Mathematics Subject Classification: 47A63, 47A99

Key words and phrases: trace class operators, Hilbert-Schmidt operators, Schwarz inequality, Grüss inequality solution 
b) Shisha-Mond's inequality [48]:

$$
\frac{\sum_{k=1}^{n} a_{k}^{2}}{\sum_{k=1}^{n} a_{k} b_{k}}-\frac{\sum_{k=1}^{n} a_{k} b_{k}}{\sum_{k=1}^{n} b_{k}^{2}} \leq\left[\left(\frac{M_{1}}{m_{2}}\right)^{\frac{1}{2}}-\left(\frac{m_{1}}{M_{2}}\right)^{\frac{1}{2}}\right]^{2} .
$$

If $\overline{\mathbf{w}}=\left(w_{1}, \ldots, w_{n}\right)$ is a positive sequence, then the following weighted inequalities also hold:

c) Cassels' inequality [15]. If the positive real sequences $\overline{\mathbf{a}}=\left(\mathrm{a}_{1}, \ldots, \mathrm{a}_{\mathrm{n}}\right)$ and $\overline{\mathbf{b}}=\left(b_{1}, \ldots, b_{n}\right)$ satisfy the condition

$$
0<m \leq \frac{a_{k}}{b_{k}} \leq M<\infty \text { for each } k \in\{1, \ldots, n\},
$$

then

$$
\frac{\left(\sum_{k=1}^{n} w_{k} a_{k}^{2}\right)\left(\sum_{k=1}^{n} w_{k} b_{k}^{2}\right)}{\left(\sum_{k=1}^{n} w_{k} a_{k} b_{k}\right)^{2}} \leq \frac{(M+m)^{2}}{4 m M} .
$$

d) Greub-Reinboldt's inequality [34]. We have

$$
\left(\sum_{k=1}^{n} w_{k} a_{k}^{2}\right)\left(\sum_{k=1}^{n} w_{k} b_{k}^{2}\right) \leq \frac{\left(M_{1} M_{2}+m_{1} m_{2}\right)^{2}}{4 m_{1} m_{2} M_{1} M_{2}}\left(\sum_{k=1}^{n} w_{k} a_{k} b_{k}\right)^{2},
$$

provided $\overline{\mathbf{a}}=\left(a_{1}, \ldots, a_{n}\right)$ and $\overline{\mathbf{b}}=\left(b_{1}, \ldots, b_{n}\right)$ satisfy the condition (1) .

For other recent results providing discrete reverse inequalities, see the monograph online [15].

The following reverse of Schwarz's inequality in inner product spaces holds [16].

Theorem 1 (Dragomir, 2003, [16]) Let $\mathrm{A}, \mathrm{a} \in \mathbb{C}$ and $x, y \in \mathrm{H}$, a complex inner product space with the inner product $\langle\cdot, \cdot\rangle$. If

$$
\operatorname{Re}\langle A y-x, x-a y\rangle \geq 0,
$$

or equivalently,

$$
\left\|x-\frac{a+A}{2} \cdot y\right\| \leq \frac{1}{2}|A-a|\|y\|,
$$

holds, then we have the inequality

$$
0 \leq\|x\|^{2}\|y\|^{2}-|\langle x, y\rangle|^{2} \leq \frac{1}{4}|A-a|^{2}\|y\|^{4} .
$$

The constant $\frac{1}{4}$ is sharp in (5). 
In 1935, G. Grüss [35] proved the following integral inequality which gives an approximation of the integral mean of the product in terms of the product of the integrals means as follows:

$$
\begin{aligned}
& \left|\frac{1}{b-a} \int_{a}^{b} f(x) g(x) d x-\frac{1}{b-a} \int_{a}^{b} f(x) d x \cdot \frac{1}{b-a} \int_{a}^{b} g(x) d x\right| \\
& \leq \frac{1}{4}(\Phi-\phi)(\Gamma-\gamma),
\end{aligned}
$$

where $f, g:[a, b] \rightarrow \mathbb{R}$ are integrable on $[a, b]$ and satisfy the condition

$$
\phi \leq f(x) \leq \Phi, \gamma \leq g(x) \leq \Gamma
$$

for each $x \in[a, b]$, where $\phi, \Phi, \gamma, \Gamma$ are given real constants.

Moreover, the constant $\frac{1}{4}$ is sharp in the sense that it cannot be replaced by a smaller one.

In [18], in order to generalize the Grüss integral inequality in abstract structures the author has proved the following inequality in inner product spaces.

Theorem 2 (Dragomir, 1999, [18]) Let $(\mathrm{H},\langle\cdot, \cdot\rangle)$ be an inner product space over $\mathbb{K}(\mathbb{K}=\mathbb{R}, \mathbb{C})$ and $e \in \mathrm{H},\|e\|=1$. If $\varphi, \gamma, \Phi, \Gamma$ are real or complex numbers and $\mathrm{x}, \mathrm{y}$ are vectors in $\mathrm{H}$ such that the conditions

$$
\operatorname{Re}\langle\Phi e-x, x-\varphi e\rangle \geq 0 \text { and } \operatorname{Re}\langle\Gamma e-y, y-\gamma e\rangle \geq 0
$$

hold, then we have the inequality

$$
|\langle x, y\rangle-\langle x, e\rangle\langle e, y\rangle| \leq \frac{1}{4}|\Phi-\varphi||\Gamma-\gamma|
$$

The constant $\frac{1}{4}$ is best possible in the sense that it can not be replaced by a smaller constant.

For other results of this type, see the recent monograph [21] and the references therein.

For other Grüss type results for integral and sums see the papers [1]-[3], [8]-[10], [17]-[24], [31], and the references therein.

In order to state some reverses of Schwarz and Grüss type inequalities for trace operators on complex Hilbert spaces we need some preparations as follows. 


\section{Some facts on trace of operators}

Let $(H,\langle\cdot, \cdot\rangle)$ be a complex Hilbert space and $\left\{\boldsymbol{e}_{\boldsymbol{i}}\right\}_{i \in \mathrm{I}}$ an orthonormal basis of $\mathrm{H}$. We say that $A \in \mathcal{B}(\mathrm{H})$ is a Hilbert-Schmidt operator if

$$
\sum_{i \in I}\left\|A e_{i}\right\|^{2}<\infty
$$

It is well know that, if $\left\{\boldsymbol{e}_{\boldsymbol{i}}\right\}_{i \in \mathrm{I}}$ and $\left\{\boldsymbol{f}_{\mathbf{j}}\right\}_{j \in \mathrm{J}}$ are orthonormal bases for $\mathrm{H}$ and $A \in \mathcal{B}(\mathrm{H})$ then

$$
\sum_{i \in I}\left\|A e_{i}\right\|^{2}=\sum_{j \in I}\left\|A f_{j}\right\|^{2}=\sum_{j \in I}\left\|A^{*} f_{j}\right\|^{2}
$$

showing that the definition (10) is independent of the orthonormal basis and $A$ is a Hilbert-Schmidt operator iff $A^{*}$ is a Hilbert-Schmidt operator.

Let $\mathcal{B}_{2}(\mathrm{H})$ the set of Hilbert-Schmidt operators in $\mathcal{B}(\mathrm{H})$. For $A \in \mathcal{B}_{2}(\mathrm{H})$ we define

$$
\|A\|_{2}:=\left(\sum_{i \in I}\left\|A e_{i}\right\|^{2}\right)^{1 / 2}
$$

for $\left\{\boldsymbol{e}_{i}\right\}_{i \in \mathrm{I}}$ an orthonormal basis of $\mathrm{H}$. This definition does not depend on the choice of the orthonormal basis.

Using the triangle inequality in $\mathrm{l}^{2}(\mathrm{I})$, one checks that $\mathcal{B}_{2}(\mathrm{H})$ is a vector space and that $\|\cdot\|_{2}$ is a norm on $\mathcal{B}_{2}(\mathrm{H})$, which is usually called in the literature as the Hilbert-Schmidt norm.

Denote the modulus of an operator $A \in \mathcal{B}(H)$ by $|A|:=\left(A^{*} A\right)^{1 / 2}$.

Because $\||A| x\|=\|A x\|$ for all $x \in H, A$ is Hilbert-Schmidt iff $|A|$ is HilbertSchmidt and $\|A\|_{2}=\||A|\|_{2}$. From (11) we have that if $A \in \mathcal{B}_{2}(H)$, then $A^{*} \in \mathcal{B}_{2}(\mathrm{H})$ and $\|A\|_{2}=\left\|A^{*}\right\|_{2}$.

If $\left\{e_{i}\right\}_{i \in I}$ an orthonormal basis of $H$, we say that $A \in \mathcal{B}(H)$ is trace class if

$$
\|A\|_{1}:=\sum_{i \in I}\left\langle|A| e_{i}, e_{i}\right\rangle<\infty .
$$

The definition of $\|A\|_{1}$ does not depend on the choice of the orthonormal basis $\left\{\boldsymbol{e}_{i}\right\}_{i \in \mathrm{I}}$. We denote by $\mathcal{B}_{1}(\mathrm{H})$ the set of trace class operators in $\mathcal{B}(\mathrm{H})$.

We define the trace of a trace class operator $A \in \mathcal{B}_{1}(\mathrm{H})$ to be

$$
\operatorname{tr}(A):=\sum_{i \in \mathrm{I}}\left\langle A e_{i}, e_{i}\right\rangle,
$$


where $\left\{e_{i}\right\}_{i \in I}$ an orthonormal basis of $H$. Note that this coincides with the usual definition of the trace if $\mathrm{H}$ is finite-dimensional. We observe that the series (14) converges absolutely and it is independent from the choice of basis.

Utilising the trace notation we obviously have that

$$
\langle A, B\rangle_{2}=\operatorname{tr}\left(B^{*} A\right)=\operatorname{tr}\left(A B^{*}\right) \text { and }\|A\|_{2}^{2}=\operatorname{tr}\left(A^{*} A\right)=\operatorname{tr}\left(|A|^{2}\right)
$$

for any $A, B \in \mathcal{B}_{2}(H)$.

The following Hölder's type inequality has been obtained by Ruskai in [45]

$$
|\operatorname{tr}(A B)| \leq \operatorname{tr}(|A B|) \leq\left[\operatorname{tr}\left(|A|^{1 / \alpha}\right)\right]^{\alpha}\left[\operatorname{tr}\left(|B|^{1 /(1-\alpha)}\right)\right]^{1-\alpha}
$$

where $\alpha \in(0,1)$ and $A, B \in \mathcal{B}(H)$ with $|A|^{1 / \alpha},|B|^{1 /(1-\alpha)} \in \mathcal{B}_{1}(H)$.

In particular, for $\alpha=\frac{1}{2}$ we get the Schwarz inequality

$$
|\operatorname{tr}(A B)| \leq \operatorname{tr}(|A B|) \leq\left[\operatorname{tr}\left(|A|^{2}\right)\right]^{1 / 2}\left[\operatorname{tr}\left(|B|^{2}\right)\right]^{1 / 2}
$$

with $A, B \in \mathcal{B}_{2}(H)$.

For the theory of trace functionals and their applications the reader is referred to [49].

For some classical trace inequalities see [11], [13], [42] and [53], which are continuations of the work of Bellman [5]. For related works the reader can refer to [4], [6], [11], [32], [36], [37], [39], [46] and [50].

We denote by

$$
\mathcal{B}_{1}^{+}(\mathrm{H}):=\left\{\mathrm{P}: \mathrm{P} \in \mathcal{B}_{1}(\mathrm{H}), \mathrm{P} \text { and is selfadjoint and } \mathrm{P} \geq 0\right\} .
$$

We obtained recently the following result [29]:

Theorem 3 For any $\mathrm{A}, \mathrm{C} \in \mathcal{B}(\mathrm{H})$ and $\mathrm{P} \in \mathcal{B}_{1}^{+}(\mathrm{H}) \backslash\{0\}$ we have the inequality

$$
\begin{aligned}
& \left|\frac{\operatorname{tr}(\mathrm{PAC})}{\operatorname{tr}(\mathrm{P})}-\frac{\operatorname{tr}(\mathrm{PA})}{\operatorname{tr}(\mathrm{P})} \frac{\operatorname{tr}(\mathrm{PC})}{\operatorname{tr}(\mathrm{P})}\right| \\
& \leq \inf _{\lambda \in \mathbb{C}}\left\|\mathrm{A}-\lambda \cdot 1_{\mathrm{H}}\right\| \frac{1}{\operatorname{tr}(\mathrm{P})} \operatorname{tr}\left(\left|\left(\mathrm{C}-\frac{\operatorname{tr}(\mathrm{PC})}{\operatorname{tr}(\mathrm{P})} 1_{\mathrm{H}}\right) \mathrm{P}\right|\right) \\
& \leq \inf _{\lambda \in \mathbb{C}}\left\|A-\lambda \cdot 1_{\mathrm{H}}\right\|\left[\frac{\operatorname{tr}\left(\mathrm{P}|\mathrm{C}|^{2}\right)}{\operatorname{tr}(\mathrm{P})}-\left|\frac{\operatorname{tr}(\mathrm{PC})}{\operatorname{tr}(\mathrm{P})}\right|^{2}\right]^{1 / 2},
\end{aligned}
$$

where $\|\cdot\|$ is the operator norm.

In the following we establish other similar results for trace that generalize the classical Cassels' inequality stated in the introduction. 


\section{Cassels type trace inequalities}

For two given operators $T, U \in B(H)$ and two given scalars $\alpha, \beta \in \mathbb{C}$ consider the transform

$$
\mathcal{C}_{\alpha, \beta}(\mathrm{T}, \mathrm{U})=\left(\mathrm{T}^{*}-\bar{\alpha} \mathrm{U}^{*}\right)(\beta \mathrm{U}-\mathrm{T}) .
$$

This transform generalizes the transform

$$
\mathcal{C}_{\alpha, \beta}(\mathrm{T}):=\left(\mathrm{T}^{*}-\bar{\alpha} 1_{\mathrm{H}}\right)\left(\beta 1_{\mathrm{H}}-\mathrm{T}\right)=\mathcal{C}_{\alpha, \beta}\left(\mathrm{T}, 1_{\mathrm{H}}\right),
$$

where $1_{\mathrm{H}}$ is the identity operator, which has been introduced in [27] in order to provide some generalizations of the well known Kantorovich inequality for operators in Hilbert spaces.

We recall that a bounded linear operator $T$ on the complex Hilbert space $(\mathrm{H},\langle\cdot, \cdot\rangle)$ is called accretive if $\operatorname{Re}\langle\mathrm{Ty}, \mathrm{y}\rangle \geq 0$ for any $\mathrm{y} \in \mathrm{H}$.

Utilizing the following identity

$$
\begin{aligned}
\operatorname{Re}\left\langle\mathcal{C}_{\alpha, \beta}(\mathrm{T}, \mathrm{U}) x, x\right\rangle & =\operatorname{Re}\left\langle\mathcal{C}_{\beta, \alpha}(\mathrm{T}, \mathrm{U}) x, x\right\rangle \\
& =\frac{1}{4}|\beta-\alpha|^{2}\|\mathrm{U} x\|^{2}-\left\|\mathrm{T} x-\frac{\alpha+\beta}{2} \cdot \mathrm{U} x\right\|^{2} \\
& =\frac{1}{4}|\beta-\alpha|^{2}\left\langle|\mathrm{U}|^{2} x, x\right\rangle-\left\langle\left|\mathrm{T}-\frac{\alpha+\beta}{2} \cdot \mathrm{U}\right|^{2} x, x\right\rangle
\end{aligned}
$$

that holds for any scalars $\alpha, \beta$ and any vector $x \in H$, we can give a simple characterization result that is useful in the following:

Lemma 1 For $\alpha, \beta \in \mathbb{C}$ and $\mathrm{T}, \mathrm{U} \in \mathrm{B}(\mathrm{H})$ the following statements are equivalent:

(i) The transform $\mathcal{C}_{\alpha, \beta}(\mathrm{T}, \mathrm{U})$ (or, equivalently, $\mathcal{C}_{\beta, \alpha}(\mathrm{T}, \mathrm{U})$ ) is accretive;

(ii) We have the norm inequality

$$
\left\|\mathrm{Tx}-\frac{\alpha+\beta}{2} \cdot \mathrm{Ux}\right\| \leq \frac{1}{2}|\beta-\alpha|\|\mathrm{U} x\|
$$

for any $\mathrm{x} \in \mathrm{H}$;

(iii) We have the following inequality in the operator order

$$
\left|\mathrm{T}-\frac{\alpha+\beta}{2} \cdot \mathrm{U}\right|^{2} \leq \frac{1}{4}|\beta-\alpha|^{2}|\mathrm{U}|^{2} .
$$


As a consequence of the above lemma we can state:

Corollary 1 Let $\alpha, \beta \in \mathbb{C}$ and $\mathrm{T}, \mathrm{U} \in \mathrm{B}(\mathrm{H})$. If $\mathcal{C}_{\alpha, \beta}(\mathrm{T}, \mathrm{U})$ is accretive, then

$$
\left\|\mathrm{T}-\frac{\alpha+\beta}{2} \cdot \mathrm{u}\right\| \leq \frac{1}{2}|\beta-\alpha|\|\mathrm{U}\|
$$

Remark 1 In order to give examples of linear operators $\mathrm{T}, \mathrm{U} \in \mathrm{B}(\mathrm{H})$ and numbers $\alpha, \beta \in \mathbb{C}$ such that the transform $\mathcal{C}_{\alpha, \beta}(\mathrm{T}, \mathrm{U})$ is accretive, it suffices to select two bounded linear operator $\mathrm{S}$ and $\mathrm{V}$ and the complex numbers $z, w$ $(w \neq 0)$ with the property that $\|\mathrm{S} x-z \mathrm{~V} x\| \leq|w|\|\mathrm{V} x\|$ for any $\mathrm{x} \in \mathrm{H}$, and, by choosing $\mathrm{T}=\mathrm{S}, \mathrm{U}=\mathrm{V}, \alpha=\frac{1}{2}(z+w)$ and $\beta=\frac{1}{2}(z-w)$ we observe that $\mathrm{T}$ and $\mathrm{U}$ satisfy $(19)$, i.e., $\mathcal{C}_{\alpha, \beta}(\mathrm{T}, \mathrm{U})$ is accretive.

The following result also holds:

Lemma 2 Let, either $\mathrm{P} \in \mathcal{B}_{+}(\mathrm{H}), \mathrm{A}, \mathrm{B} \in \mathcal{B}_{2}(\mathrm{H})$ or $\mathrm{P} \in \mathcal{B}_{1}^{+}(\mathrm{H}), \mathrm{A}, \mathrm{B} \in$ $\mathcal{B}(\mathrm{H})$ and $\gamma, \Gamma \in \mathbb{C}$. Then

$$
\operatorname{Re}\left(\operatorname{tr}\left[\mathrm{P}\left(\mathrm{A}^{*}-\bar{\gamma} \mathrm{B}^{*}\right)(\Gamma \mathrm{B}-\mathrm{A})\right]\right) \geq 0
$$

if and only if

$$
\operatorname{tr}\left(\mathrm{P}\left|\mathrm{A}-\frac{\gamma+\Gamma}{2} \mathrm{~B}\right|^{2}\right) \leq \frac{1}{4}|\Gamma-\gamma|^{2} \operatorname{tr}\left(\mathrm{P}|\mathrm{B}|^{2}\right) .
$$

To simplify the writing, we the say that $(\mathrm{A}, \mathrm{B})$ satisfies the $\mathrm{P}-(\gamma, \Gamma)$-trace property.

Proof. We have the equalities

$$
\begin{aligned}
& \frac{1}{4}|\Gamma-\gamma|^{2} \mathrm{P}|\mathrm{B}|^{2}-\mathrm{P}\left|\mathrm{A}-\frac{\gamma+\Gamma}{2} \mathrm{~B}\right|^{2} \\
& =\mathrm{P}\left[\frac{1}{4}|\Gamma-\gamma|^{2}|\mathrm{~B}|^{2}-\left|\mathrm{A}-\frac{\gamma+\Gamma}{2} \mathrm{~B}\right|^{2}\right] \\
& =\mathrm{P}\left[\frac{1}{4}|\Gamma-\gamma|^{2}|\mathrm{~B}|^{2}-\left(\mathrm{A}-\frac{\gamma+\Gamma}{2} \mathrm{~B}\right)^{*}\left(\mathrm{~A}-\frac{\gamma+\Gamma}{2} \mathrm{~B}\right)\right]
\end{aligned}
$$




$$
\begin{aligned}
& =\mathrm{P}\left[\frac{1}{4}|\Gamma-\gamma|^{2}|\mathrm{~B}|^{2}-|\mathrm{A}|^{2}+\frac{\overline{\gamma+\Gamma}}{2} \mathrm{~B}^{*} \mathrm{~A}+\frac{\gamma+\Gamma}{2} \mathrm{~A}^{*} \mathrm{~B}-\left|\frac{\gamma+\Gamma}{2}\right|^{2}|\mathrm{~B}|^{2}\right] \\
& =\mathrm{P}\left[-|\mathrm{A}|^{2}+\frac{\overline{\gamma+\Gamma}}{2} \mathrm{~B}^{*} \mathrm{~A}+\frac{\gamma+\Gamma}{2} \mathrm{~A}^{*} \mathrm{~B}+\left(\frac{1}{4}|\Gamma-\gamma|^{2}-\left|\frac{\gamma+\Gamma}{2}\right|^{2}\right)|\mathrm{B}|^{2}\right] \\
& =\mathrm{P}\left[-|\mathrm{A}|^{2}+\frac{\overline{\gamma+\Gamma}}{2} \mathrm{~B}^{*} \mathrm{~A}+\frac{\gamma+\Gamma}{2} \mathrm{~A}^{*} \mathrm{~B}-\operatorname{Re}(\Gamma \bar{\gamma})|\mathrm{B}|^{2}\right]
\end{aligned}
$$

for any bounded operators $A, B, P$ and the complex numbers $\gamma, \Gamma \in \mathbb{C}$.

Taking the trace in (23) we get

$$
\begin{aligned}
& \frac{1}{4}|\Gamma-\gamma|^{2} \operatorname{tr}\left(\mathrm{P}|\mathrm{B}|^{2}\right)-\operatorname{tr}\left(\mathrm{P}\left|\mathrm{A}-\frac{\gamma+\Gamma}{2} \mathrm{~B}\right|^{2}\right) \\
& =-\operatorname{tr}\left(\mathrm{P}|\mathrm{A}|^{2}\right)-\operatorname{Re}(\Gamma \bar{\gamma}) \operatorname{tr}\left(\mathrm{P}|\mathrm{B}|^{2}\right)+\frac{\overline{\gamma+\Gamma}}{2} \operatorname{tr}\left(\mathrm{PB}^{*} \mathrm{~A}\right)+\frac{\gamma+\Gamma}{2} \operatorname{tr}\left(\mathrm{PA}^{*} \mathrm{~B}\right) \\
& =-\operatorname{tr}\left(\mathrm{P}|\mathrm{A}|^{2}\right)-\operatorname{Re}(\Gamma \bar{\gamma}) \operatorname{tr}\left(\mathrm{P}|\mathrm{B}|^{2}\right)+\frac{\overline{\gamma+\Gamma}}{2} \operatorname{tr}\left(\mathrm{PB}^{*} \mathrm{~A}\right)+\frac{\gamma+\Gamma \overline{\operatorname{tr}\left(\mathrm{PB}^{*} \mathrm{~A}\right)}}{2} \\
& =-\operatorname{tr}\left(\mathrm{P}|\mathrm{A}|^{2}\right)-\operatorname{Re}(\Gamma \bar{\gamma}) \operatorname{tr}\left(\mathrm{P}|\mathrm{B}|^{2}\right)+\overline{\frac{\gamma+\Gamma}{2}} \operatorname{tr}\left(\mathrm{PB}^{*} \mathrm{~A}\right)+\overline{\overline{\frac{\gamma+\Gamma}{2}}} \operatorname{tr}\left(\mathrm{PB}^{*} \mathrm{~A}\right) \\
& =-\operatorname{tr}\left(\mathrm{P}|\mathrm{A}|^{2}\right)-\operatorname{Re}(\Gamma \bar{\gamma}) \operatorname{tr}\left(\mathrm{P}|\mathrm{B}|^{2}\right)+2 \operatorname{Re}\left[\frac{\overline{\gamma+\Gamma}}{2} \operatorname{tr}\left(\mathrm{PB}^{*} \mathrm{~A}\right)\right] \\
& =-\operatorname{tr}\left(\mathrm{P}|\mathrm{A}|^{2}\right)-\operatorname{Re}(\Gamma \bar{\gamma}) \operatorname{tr}\left(\mathrm{P}|\mathrm{B}|^{2}\right)+\operatorname{Re}\left[\bar{\gamma} \operatorname{tr}\left(\mathrm{PB}^{*} \mathrm{~A}\right)\right]+\operatorname{Re}\left[\bar{\Gamma} \operatorname{tr}\left(\mathrm{PB}^{*} \mathrm{~A}\right)\right] \\
& =-\operatorname{tr}\left(\mathrm{P}|\mathrm{A}|^{2}\right)-\operatorname{Re}(\Gamma \bar{\gamma}) \operatorname{tr}\left(\mathrm{P}|\mathrm{B}|^{2}\right)+\operatorname{Re}\left[\bar{\gamma} \operatorname{tr}\left(\mathrm{PB}^{*} \mathrm{~A}\right)\right]+\operatorname{Re}\left[\overline{\left.\overline{\Gamma \operatorname{tr}\left(\mathrm{PB}^{*} \mathrm{~A}\right)}\right]}\right. \\
& =-\operatorname{tr}\left(\mathrm{P}|\mathrm{A}|^{2}\right)-\operatorname{Re}(\Gamma \bar{\gamma}) \operatorname{tr}\left(\mathrm{P}|\mathrm{B}|^{2}\right)+\operatorname{Re}\left[\bar{\gamma} \operatorname{tr}\left(\mathrm{PB}^{*} \mathrm{~A}\right)\right]+\operatorname{Re}\left[\Gamma \overline{\operatorname{tr}\left(\mathrm{PB}^{*} \mathrm{~A}\right)}\right] .
\end{aligned}
$$

Since

$$
\begin{aligned}
& \operatorname{Re}\left(\operatorname{tr}\left[\mathrm{P}\left(\mathrm{A}^{*}-\bar{\gamma} \mathrm{B}^{*}\right)(\Gamma \mathrm{B}-\mathrm{A})\right]\right) \\
& =\operatorname{Re}\left[\operatorname{tr}\left(\Gamma \mathrm{P} \mathrm{A}^{*} \mathrm{~B}+\bar{\gamma} \mathrm{PB}^{*} \mathrm{~A}-\bar{\gamma} \Gamma \mathrm{PB}^{*} \mathrm{~B}-\mathrm{PA}^{*} \mathrm{~A}\right)\right] \\
& \left.=\operatorname{Re}\left[\Gamma \operatorname{tr}\left(\mathrm{P} A^{*} \mathrm{~B}\right)+\bar{\gamma} \operatorname{tr}\left(\mathrm{PB}^{*} \mathrm{~A}\right)\right]-\bar{\gamma} \Gamma \operatorname{tr}\left(\mathrm{P}|\mathrm{B}|^{2}\right)-\operatorname{tr}\left(\mathrm{P}|\mathrm{A}|^{2}\right)\right] \\
& =\operatorname{Re}\left[\overline{\operatorname{tr}\left(\mathrm{PB} \mathrm{B}^{*}\right)}+\bar{\gamma} \operatorname{tr}\left(\mathrm{PB} \mathrm{B}^{*} \mathrm{~A}\right)\right]-\operatorname{tr}\left(\mathrm{P}|\mathrm{B}|^{2}\right) \operatorname{Re}(\bar{\gamma} \Gamma)-\operatorname{tr}\left(\mathrm{P}|\mathrm{A}|^{2}\right),
\end{aligned}
$$

then we get

$$
\begin{aligned}
& \frac{1}{4}|\Gamma-\gamma|^{2} \operatorname{tr}\left(\mathrm{P}|\mathrm{B}|^{2}\right)-\operatorname{tr}\left(\mathrm{P}\left|\mathrm{A}-\frac{\gamma+\Gamma}{2} \mathrm{~B}\right|^{2}\right) \\
& =\operatorname{Re}\left(\operatorname{tr}\left[\mathrm{P}\left(\mathrm{A}^{*}-\bar{\gamma} \mathrm{B}^{*}\right)(\Gamma \mathrm{B}-\mathrm{A})\right]\right),
\end{aligned}
$$


which proves the desired equivalence.

Corollary 2 Let, either $\mathrm{P} \in \mathcal{B}_{+}(\mathrm{H}), \mathrm{A}, \mathrm{B} \in \mathcal{B}_{2}(\mathrm{H})$ or $\mathrm{P} \in \mathcal{B}_{1}^{+}(\mathrm{H}), \mathrm{A}$, $\mathrm{B} \in \mathcal{B}(\mathrm{H})$ and $\gamma, \Gamma \in \mathbb{C}$. If the transform $\mathcal{C}_{\gamma, \Gamma}(\mathrm{A}, \mathrm{B})$ is accretive, then $(\mathrm{A}, \mathrm{B})$ satisfies the $\mathrm{P}-(\gamma, \Gamma)$-trace property.

We have the following result:

Theorem 4 Let, either $\mathrm{P} \in \mathcal{B}_{+}(\mathrm{H}), \mathrm{A}, \mathrm{B} \in \mathcal{B}_{2}(\mathrm{H})$ or $\mathrm{P} \in \mathcal{B}_{1}^{+}(\mathrm{H}), \mathrm{A}, \mathrm{B} \in$ $\mathcal{B}(\mathrm{H})$ and $\gamma, \Gamma \in \mathbb{C}$ with $\operatorname{Re}(\Gamma \bar{\gamma})=\operatorname{Re}(\Gamma) \operatorname{Re}(\gamma)+\operatorname{Im}(\Gamma) \operatorname{Im}(\gamma)>0$.

(i) If $(\mathrm{A}, \mathrm{B})$ satisfies the $\mathrm{P}-(\gamma, \Gamma)$-trace property, then we have

$$
\begin{aligned}
& \operatorname{tr}\left(\mathrm{P}|\mathrm{A}|^{2}\right) \operatorname{tr}\left(\mathrm{P}|\mathrm{B}|^{2}\right) \\
& \leq \frac{1}{4} \cdot \frac{\left[\operatorname{Re}(\gamma+\Gamma) \operatorname{Re} \operatorname{tr}\left(\mathrm{PB}^{*} \mathrm{~A}\right)+\operatorname{Im}(\gamma+\Gamma) \operatorname{Im} \operatorname{tr}\left(\mathrm{PB}^{*} \mathrm{~A}\right)\right]^{2}}{\operatorname{Re}(\Gamma) \operatorname{Re}(\gamma)+\operatorname{Im}(\Gamma) \operatorname{Im}(\gamma)} \\
& \leq \frac{1}{4} \cdot \frac{|\gamma+\Gamma|^{2}}{\operatorname{Re}(\Gamma \bar{\gamma})}\left|\operatorname{tr}\left(\mathrm{PB}^{*} \mathrm{~A}\right)\right|^{2} .
\end{aligned}
$$

(ii) If the transform $\mathcal{C}_{\gamma, \Gamma}(\mathrm{A}, \mathrm{B})$ is accretive, then the inequality (26) also holds.

Proof. (i) If $(A, B)$ satisfies the $\mathrm{P}-(\gamma, \Gamma)$-trace property, then, on utilizing the calculations above, we have

$$
\begin{aligned}
& 0 \leq \frac{1}{4}|\Gamma-\gamma|^{2} \operatorname{tr}\left(\mathrm{P}|\mathrm{B}|^{2}\right)-\operatorname{tr}\left(\mathrm{P}\left|\mathrm{A}-\frac{\gamma+\Gamma}{2} \mathrm{~B}\right|^{2}\right) \\
& =-\operatorname{tr}\left(\mathrm{P}|\mathrm{A}|^{2}\right)-\operatorname{Re}(\Gamma \bar{\gamma}) \operatorname{tr}\left(\mathrm{P}|\mathrm{B}|^{2}\right)+\operatorname{Re}\left[\bar{\gamma} \operatorname{tr}\left(\mathrm{PB}^{*} \mathrm{~A}\right)\right]+\operatorname{Re}\left[\Gamma \overline{\operatorname{tr}\left(\mathrm{PB}^{*} \mathrm{~A}\right)}\right] \\
& =-\operatorname{tr}\left(\mathrm{P}|\mathrm{A}|^{2}\right)-\operatorname{Re}(\Gamma \bar{\gamma}) \operatorname{tr}\left(\mathrm{P}|\mathrm{B}|^{2}\right)+\operatorname{Re}\left[\bar{\gamma} \operatorname{tr}\left(\mathrm{PB}^{*} \mathrm{~A}\right)\right]+\operatorname{Re}\left[\overline{\overline{\Gamma \operatorname{tr}\left(\mathrm{PB}^{*} \mathrm{~A}\right)}}\right] \\
& =-\operatorname{tr}\left(\mathrm{P}|\mathrm{A}|^{2}\right)-\operatorname{Re}(\Gamma \bar{\gamma}) \operatorname{tr}\left(\mathrm{P}|\mathrm{B}|^{2}\right)+\operatorname{Re}\left[\bar{\gamma} \operatorname{tr}\left(\mathrm{PB}^{*} \mathrm{~A}\right)\right]+\operatorname{Re}\left[\bar{\Gamma} \operatorname{tr}\left(\mathrm{PB}^{*} \mathrm{~A}\right)\right] \\
& =-\operatorname{tr}\left(\mathrm{P}|\mathrm{A}|^{2}\right)-\operatorname{Re}(\Gamma \bar{\gamma}) \operatorname{tr}\left(\mathrm{P}|\mathrm{B}|^{2}\right)+\operatorname{Re}\left[(\bar{\gamma}+\bar{\Gamma}) \operatorname{tr}\left(\mathrm{PB}^{*} \mathrm{~A}\right)\right] \text {, }
\end{aligned}
$$

which implies that

$$
\begin{aligned}
& \operatorname{tr}\left(\mathrm{P}|\mathrm{A}|^{2}\right)+\operatorname{Re}(\Gamma \bar{\gamma}) \operatorname{tr}\left(\mathrm{P}|\mathrm{B}|^{2}\right) \leq \operatorname{Re}\left[(\bar{\gamma}+\bar{\Gamma}) \operatorname{tr}\left(\mathrm{PB}^{*} \mathrm{~A}\right)\right] \\
& =\operatorname{Re}(\gamma+\Gamma) \operatorname{Re} \operatorname{tr}\left(\mathrm{PB}^{*} \mathrm{~A}\right)+\operatorname{Im}(\gamma+\Gamma) \operatorname{Im} \operatorname{tr}\left(\mathrm{PB}^{*} \mathrm{~A}\right) .
\end{aligned}
$$

Making use of the elementary inequality

$$
2 \sqrt{p q} \leq p+q, p, q \geq 0,
$$


we also have

$$
2 \sqrt{\operatorname{Re}(\Gamma \bar{\gamma}) \operatorname{tr}\left(\mathrm{P}|\mathrm{A}|^{2}\right) \operatorname{tr}\left(\mathrm{P}|\mathrm{B}|^{2}\right)} \leq \operatorname{tr}\left(\mathrm{P}|\mathrm{A}|^{2} \text { big }\right)+\operatorname{Re}(\Gamma \bar{\gamma}) \operatorname{tr}\left(\mathrm{P}|\mathrm{B}|^{2}\right) .
$$

Utilising (27) and (28) we get

$$
\begin{aligned}
& \sqrt{\operatorname{tr}\left(\mathrm{P}|\mathrm{A}|^{2}\right) \operatorname{tr}\left(\mathrm{P}|\mathrm{B}|^{2}\right)} \\
& \leq \frac{\operatorname{Re}(\gamma+\Gamma) \operatorname{Re} \operatorname{tr}\left(\mathrm{PB}^{*} \mathrm{~A}\right)+\operatorname{Im}(\gamma+\Gamma) \operatorname{Im} \operatorname{tr}\left(\mathrm{PB}^{*} \mathrm{~A}\right)}{2 \sqrt{\operatorname{Re}(\Gamma \bar{\gamma})}}
\end{aligned}
$$

that is equivalent with the first inequality in (26).

The second inequality in (26) is obvious by Schwarz inequality

$$
(a b+c d)^{2} \leq\left(a^{2}+c^{2}\right)\left(b^{2}+d^{2}\right), a, b, c, d \in \mathbb{R} .
$$

The (ii) is obvious from (i).

Remark 2 We observe that the inequality between the first and last term in (26) is equivalent to

$$
0 \leq \operatorname{tr}\left(\mathrm{P}|\mathrm{A}|^{2}\right) \operatorname{tr}\left(\mathrm{P}|\mathrm{B}|^{2}\right)-\left|\operatorname{tr}\left(\mathrm{PB}^{*} \mathrm{~A}\right)\right|^{2} \leq \frac{1}{4} \cdot \frac{|\gamma-\Gamma|^{2}}{\operatorname{Re}(\Gamma \bar{\gamma})}\left|\operatorname{tr}\left(\mathrm{PB}^{*} \mathrm{~A}\right)\right|^{2} .
$$

Corollary 3 Let, either $\mathrm{P} \in \mathcal{B}_{+}(\mathrm{H}), \mathrm{A} \in \mathcal{B}_{2}(\mathrm{H})$ or $\mathrm{P} \in \mathcal{B}_{1}^{+}(\mathrm{H}), \mathrm{A} \in \mathcal{B}(\mathrm{H})$ and $\gamma, \Gamma \in \mathbb{C}$ with $\operatorname{Re}(\Gamma \bar{\gamma})=\operatorname{Re}(\Gamma) \operatorname{Re}(\gamma)+\operatorname{Im}(\Gamma) \operatorname{Im}(\gamma)>0$.

(i) If A satisfies the $\mathrm{P}-(\gamma, \Gamma)$-trace property, namely

$$
\operatorname{Re}\left(\operatorname{tr}\left[\mathrm{P}\left(\mathrm{A}^{*}-\bar{\gamma} 1_{\mathrm{H}}\right)\left(\Gamma 1_{\mathrm{H}}-\mathrm{A}\right)\right]\right) \geq 0
$$

or, equivalently

$$
\operatorname{tr}\left(\mathrm{P}\left|A-\frac{\gamma+\Gamma}{2} 1_{\mathrm{H}}\right|^{2}\right) \leq \frac{1}{4}|\Gamma-\gamma|^{2} \operatorname{tr}(\mathrm{P}),
$$

then we have

$$
\begin{aligned}
& \frac{\operatorname{tr}\left(\mathrm{P}|\mathrm{A}|^{2}\right)}{\operatorname{tr}(\mathrm{P})} \leq \frac{1}{4} \cdot \frac{\left[\operatorname{Re}(\gamma+\Gamma) \frac{\operatorname{Retr}(\mathrm{PA})}{\operatorname{tr}(\mathrm{P})}+\operatorname{Im}(\gamma+\Gamma) \frac{\operatorname{Imtr}(\mathrm{PA})}{\operatorname{tr}(\mathrm{P})}\right]^{2}}{\operatorname{Re}(\Gamma) \operatorname{Re}(\gamma)+\operatorname{Im}(\Gamma) \operatorname{Im}(\gamma)} \\
& \leq \frac{1}{4} \cdot \frac{|\gamma+\Gamma|^{2}}{\operatorname{Re}(\Gamma \bar{\gamma})}\left|\frac{\operatorname{tr}(\mathrm{PA})}{\operatorname{tr}(\mathrm{P})}\right|^{2} .
\end{aligned}
$$


(ii) If the transform $\mathcal{C}_{\gamma, \Gamma}(\mathrm{A})$ is accretive, then the inequality (26) also holds.

(iii) We have

$$
0 \leq \frac{\operatorname{tr}\left(\mathrm{P}|\mathrm{A}|^{2}\right)}{\operatorname{tr}(\mathrm{P})}-\left|\frac{\operatorname{tr}(\mathrm{PA})}{\operatorname{tr}(\mathrm{P})}\right|^{2} \leq \frac{1}{4} \cdot \frac{|\gamma-\Gamma|^{2}}{\operatorname{Re}(\Gamma \bar{\gamma})}\left|\frac{\operatorname{tr}(\mathrm{PA})}{\operatorname{tr}(\mathrm{P})}\right|^{2} .
$$

Remark 3 The case of selfadjoint operators is as follows.

Let $\mathrm{A}, \mathrm{B}$ be selfadjoint operators and either $\mathrm{P} \in \mathcal{B}_{+}(\mathrm{H}), \mathrm{A}, \mathrm{B} \in \mathcal{B}_{2}(\mathrm{H})$ or $\mathrm{P} \in \mathcal{B}_{1}^{+}(\mathrm{H}), \mathrm{A}, \mathrm{B} \in \mathcal{B}(\mathrm{H})$ and $\mathrm{m}, \mathrm{M} \in \mathbb{R}$ with $\mathrm{mM}>0$.

(i) If $(\mathrm{A}, \mathrm{B})$ satisfies the $\mathrm{P}-(\mathrm{m}, \mathrm{M})$-trace property, then we have

$$
\operatorname{tr}\left(\mathrm{PA}^{2}\right) \operatorname{tr}\left(\mathrm{PB}^{2}\right) \leq \frac{(\mathrm{m}+\mathrm{M})^{2}}{4 \mathrm{mM}}[\operatorname{tr}(\mathrm{PBA})]^{2}
$$

or, equivalently

$$
0 \leq \operatorname{tr}\left(\mathrm{PA}^{2}\right) \operatorname{tr}\left(\mathrm{PB}^{2}\right)-[\operatorname{tr}(\mathrm{PBA})]^{2} \leq \frac{(\mathrm{m}-\mathrm{M})^{2}}{4 m \mathrm{M}}[\operatorname{tr}(\mathrm{PBA})]^{2} .
$$

(ii) If the transform $\mathcal{C}_{\mathrm{m}, \mathrm{M}}(\mathrm{A}, \mathrm{B})$ is accretive, then the inequality (35) also holds.

(iii) If $(\mathrm{A}-\mathrm{mB})(\mathrm{MB}-\mathrm{A}) \geq 0$, then (35) is valid.

We observe that the inequality (35) is the operator trace inequality version of Cassels' inequality from Introduction.

\section{Trace inequalities of Grüss type}

Let $\mathrm{P}$ be a selfadjoint operator with $\mathrm{P} \geq 0$. The functional $\langle\cdot, \cdot\rangle_{2, \mathrm{P}}$ defined by

$$
\langle\mathrm{A}, \mathrm{B}\rangle_{2, \mathrm{P}}:=\operatorname{tr}\left(\mathrm{PB}^{*} \mathrm{~A}\right)=\operatorname{tr}\left(\mathrm{APB}^{*}\right)=\operatorname{tr}\left(\mathrm{B}^{*} \mathrm{AP}\right)
$$

is a nonnegative Hermitian form on $\mathcal{B}_{2}(\mathrm{H})$, i.e. $\langle\cdot, \cdot\rangle_{2, \mathrm{P}}$ satisfies the properties:

(h) $\langle A, A\rangle_{2, P} \geq 0$ for any $A \in \mathcal{B}_{2}(H)$;

(hh) $\langle\cdot, \cdot\rangle_{2, \mathrm{P}}$ is linear in the first variable;

(hhh) $\langle\mathrm{B}, \mathrm{A}\rangle_{2, \mathrm{P}}=\overline{\langle A, \mathrm{~B}\rangle}_{2, \mathrm{P}}$ for any $\mathrm{A}, \mathrm{B} \in \mathcal{B}_{2}(\mathrm{H})$.

Using the properties of the trace we also have the following representations

$$
\|A\|_{2, P}^{2}:=\operatorname{tr}\left(P|A|^{2}\right)=\operatorname{tr}\left(A P A^{*}\right)=\operatorname{tr}\left(|A|^{2} P\right)
$$

and

$$
\langle A, B\rangle_{2, P}=\operatorname{tr}\left(A P B^{*}\right)=\operatorname{tr}\left(B^{*} A P\right)
$$

for any $A, B \in \mathcal{B}_{2}(H)$.

The same definitions can be considered if $\mathrm{P} \in \mathcal{B}_{1}^{+}(\mathrm{H})$ and $A, B \in \mathcal{B}(\mathrm{H})$.

We have the following Grüss type inequality: 
Theorem 5 Let, either $\mathrm{P} \in \mathcal{B}_{+}(\mathrm{H}), \mathrm{A}, \mathrm{B}, \mathrm{C} \in \mathcal{B}_{2}(\mathrm{H})$ or $\mathrm{P} \in \mathcal{B}_{1}^{+}(\mathrm{H}), \mathrm{A}$, $\mathrm{B}, \mathrm{C} \in \mathcal{B}(\mathrm{H})$ with $\mathrm{P}|\mathrm{A}|^{2}, \mathrm{P}|\mathrm{B}|^{2}, \mathrm{P}|\mathrm{C}|^{2} \neq 0$ and $\lambda, \Gamma, \delta, \Delta \in \mathbb{C}$ with $\operatorname{Re}(\Gamma \bar{\gamma})$, $\operatorname{Re}(\Delta \bar{\delta})>0$. If $(\mathrm{A}, \mathrm{C})$ has the trace $\mathrm{P}-(\lambda, \Gamma)$-property and $(\mathrm{B}, \mathrm{C})$ has the trace $\mathrm{P}-(\delta, \Delta)$-property, then

$$
\left|\frac{\operatorname{tr}\left(\mathrm{PB}^{*} \mathrm{~A}\right) \operatorname{tr}\left(\mathrm{P}|\mathrm{C}|^{2}\right)}{\operatorname{tr}(\mathrm{PC} * \mathrm{~A}) \operatorname{tr}\left(\mathrm{PB} \mathrm{C}^{*}\right)}-1\right| \leq \frac{1}{4} \cdot \frac{|\gamma-\Gamma||\delta-\Delta|}{\sqrt{\operatorname{Re}(\Gamma \bar{\gamma}) \operatorname{Re}(\Delta \bar{\delta})}} .
$$

Proof. We prove in the case that $\mathrm{P} \in \mathcal{B}_{+}(\mathrm{H})$ and $\mathrm{A}, \mathrm{B}, \mathrm{C} \in \mathcal{B}_{2}(\mathrm{H})$.

Making use of the Schwarz inequality for the nonnegative hermitian form $\langle\cdot, \cdot\rangle_{2, \mathrm{P}}$ we have

$$
\left|\langle A, B\rangle_{2, P}\right|^{2} \leq\langle A, A\rangle_{2, P}\langle B, B\rangle_{2, P}
$$

for any $A, B \in \mathcal{B}_{2}(H)$.

Let $\mathrm{C} \in \mathcal{B}_{2}(\mathrm{H}), \mathrm{C} \neq 0$. Define the mapping $[\cdot, \cdot]_{2, \mathrm{P}, \mathrm{C}}: \mathcal{B}_{2}(\mathrm{H}) \times \mathcal{B}_{2}(\mathrm{H}) \rightarrow \mathbb{C}$ by

$$
[A, B]_{2, P, C}:=\langle A, B\rangle_{2, P}\|C\|_{2, P}^{2}-\langle A, C\rangle_{2, P}\langle C, B\rangle_{2, P} .
$$

Observe that $[\cdot, \cdot]_{2, P, C}$ is a nonnegative Hermitian form on $\mathcal{B}_{2}(\mathrm{H})$ and by Schwarz inequality we also have

$$
\begin{aligned}
& \left|\langle A, B\rangle_{2, P}\|C\|_{2, P}^{2}-\langle A, C\rangle_{2, P}\langle C, B\rangle_{2, P}\right|^{2} \\
& \leq\left[\|A\|_{2, P}^{2}\|C\|_{2, P}^{2}-\left|\langle A, C\rangle_{2, P}\right|^{2}\right]\left[\|B\|_{2, P}^{2}\|C\|_{2, P}^{2}-\left|\langle B, C\rangle_{2, P}\right|^{2}\right]
\end{aligned}
$$

for any $A, B \in \mathcal{B}_{2}(H)$, namely

$$
\begin{aligned}
& \left|\operatorname{tr}\left(\mathrm{PB}^{*} \mathrm{~A}\right) \operatorname{tr}\left(\mathrm{P}|\mathrm{C}|^{2}\right)-\operatorname{tr}\left(\mathrm{PC}^{*} \mathrm{~A}\right) \operatorname{tr}\left(\mathrm{PB}^{*} \mathrm{C}\right)\right|^{2} \\
& \leq\left[\operatorname{tr}\left(\mathrm{P}|\mathrm{A}|^{2}\right) \operatorname{tr}\left(\mathrm{P}|\mathrm{C}|^{2}\right)-\left|\operatorname{tr}\left(\mathrm{PC}^{*} \mathrm{~A}\right)\right|^{2}\right] \\
& \times\left[\operatorname{tr}\left(\mathrm{P}|\mathrm{B}|^{2}\right) \operatorname{tr}\left(\mathrm{P}|\mathrm{C}|^{2}\right)-\left|\operatorname{tr}\left(\mathrm{PB}^{*} \mathrm{C}\right)\right|^{2}\right]
\end{aligned}
$$

where for the last term we used the equality $\left|\langle B, C\rangle_{2, P}\right|^{2}=\left|\langle C, B\rangle_{2, P}\right|^{2}$.

Since $(A, C)$ has the trace $P-(\lambda, \Gamma)$-property and $(B, C)$ has the trace $P-(\delta, \Delta)$ -property, then by (30) we have

$$
0 \leq \operatorname{tr}\left(\mathrm{P}|\mathrm{A}|^{2}\right) \operatorname{tr}\left(\mathrm{P}|\mathrm{C}|^{2}\right)-\left|\operatorname{tr}\left(\mathrm{PC} \mathrm{C}^{*} \mathrm{~A}\right)\right|^{2} \leq \frac{1}{4} \cdot \frac{|\gamma-\Gamma|^{2}}{\operatorname{Re}(\Gamma \bar{\gamma})}|\operatorname{tr}(\mathrm{PC} * \mathrm{~A})|^{2}
$$


and

$$
0 \leq \operatorname{tr}\left(\mathrm{P}|\mathrm{B}|^{2}\right) \operatorname{tr}\left(\mathrm{P}|\mathrm{C}|^{2}\right)-\left|\operatorname{tr}\left(\mathrm{PB}^{*} \mathrm{C}\right)\right|^{2} \leq \frac{1}{4} \cdot \frac{|\delta-\Delta|^{2}}{\operatorname{Re}(\Delta \bar{\delta})}\left|\operatorname{tr}\left(\mathrm{PB}^{*} \mathrm{C}\right)\right|^{2} .
$$

If we multiply the inequalities (39) and (40) we get

$$
\begin{aligned}
& {\left[\operatorname{tr}\left(\mathrm{P}|\mathrm{A}|^{2}\right) \operatorname{tr}\left(\mathrm{P}|\mathrm{C}|^{2}\right)-\left|\operatorname{tr}\left(\mathrm{PC}^{*} \mathrm{~A}\right)\right|^{2}\right]} \\
& \times\left[\operatorname{tr}\left(\mathrm{P}|\mathrm{B}|^{2}\right) \operatorname{tr}\left(\mathrm{P}|\mathrm{C}|^{2}\right)-\left|\operatorname{tr}\left(\mathrm{PB}^{*} \mathrm{C}\right)\right|^{2}\right] \\
& \leq \frac{1}{16} \cdot \frac{|\gamma-\Gamma|^{2}}{\operatorname{Re}(\Gamma \bar{\gamma})} \frac{|\delta-\Delta|^{2}}{\operatorname{Re}(\Delta \bar{\delta})}\left|\operatorname{tr}\left(\mathrm{PC}^{*} \mathrm{~A}\right)\right|^{2}\left|\operatorname{tr}\left(\mathrm{PB}^{*} \mathrm{C}\right)\right|^{2} .
\end{aligned}
$$

If we use (38) and (41) we get

$$
\begin{aligned}
& \left|\operatorname{tr}\left(\mathrm{PB}^{*} \mathrm{~A}\right) \operatorname{tr}\left(\mathrm{P}|\mathrm{C}|^{2}\right)-\operatorname{tr}\left(\mathrm{PC}^{*} \mathrm{~A}\right) \operatorname{tr}\left(\mathrm{PB}^{*} \mathrm{C}\right)\right|^{2} \\
& \leq \frac{1}{16} \cdot \frac{|\gamma-\Gamma|^{2}}{\operatorname{Re}(\Gamma \bar{\gamma})} \frac{|\delta-\Delta|^{2}}{\operatorname{Re}(\Delta \bar{\delta})}\left|\operatorname{tr}\left(\mathrm{PC}^{*} \mathrm{~A}\right)\right|^{2}\left|\operatorname{tr}\left(\mathrm{PB}^{*} \mathrm{C}\right)\right|^{2} .
\end{aligned}
$$

Since $P, A, B, C \neq 0$ then by (39) and (40) we get $\operatorname{tr}\left(P^{*} A\right) \neq 0$ and $\operatorname{tr}\left(\mathrm{PB}^{*} \mathrm{C}\right) \neq 0$. Now, if we take the square root in $(42)$ and divide by $\mid \operatorname{tr}\left(\mathrm{PC}^{*} \mathrm{~A}\right)$ $\operatorname{tr}\left(\mathrm{PB}^{*} \mathrm{C}\right) \mid$ we obtain the desired result (37).

Corollary 4 Let, either $\mathrm{P} \in \mathcal{B}_{+}(\mathrm{H}), \mathrm{A}, \mathrm{B} \in \mathcal{B}_{2}$ or $\mathrm{P} \in \mathcal{B}_{1}^{+}(\mathrm{H}), \mathrm{A}, \mathrm{B} \in \mathcal{B}(\mathrm{H})$ with $\mathrm{P}|\mathrm{A}|^{2}, \mathrm{P}|\mathrm{B}|^{2} \neq 0$ and $\lambda, \Gamma, \delta, \Delta \in \mathbb{C}$ with $\operatorname{Re}(\Gamma \bar{\gamma}), \operatorname{Re}(\Delta \bar{\delta})>0$. If $\mathrm{A}$ has the trace $\mathrm{P}-(\lambda, \Gamma)$-property and $\mathrm{B}$ has the trace $\mathrm{P}-(\delta, \Delta)$-property, then

$$
\left|\frac{\operatorname{tr}\left(\mathrm{PB}^{*} \mathrm{~A}\right) \operatorname{tr}(\mathrm{P})}{\operatorname{tr}(\mathrm{PA}) \operatorname{tr}\left(\mathrm{PB}^{*}\right)}-1\right| \leq \frac{1}{4} \cdot \frac{|\gamma-\Gamma||\delta-\Delta|}{\sqrt{\operatorname{Re}(\Gamma \bar{\gamma}) \operatorname{Re}(\Delta \bar{\delta})}} .
$$

The case of selfadjoint operators is useful for applications.

Remark 4 Assume that $\mathrm{A}, \mathrm{B}, \mathrm{C}$ are selfadjoint operators. If, either $\mathrm{P} \in$ $\mathcal{B}_{+}(\mathrm{H}), \mathrm{A}, \mathrm{B}, \mathrm{C} \in \mathcal{B}_{2}(\mathrm{H})$ or $\mathrm{P} \in \mathcal{B}_{1}^{+}(\mathrm{H}), \mathrm{A}, \mathrm{B}, \mathrm{C} \in \mathcal{B}(\mathrm{H})$ with $\mathrm{PA}^{2}, \mathrm{~PB}^{2}$, $\mathrm{PC}^{2} \neq 0$ and $\mathrm{m}, \mathrm{M}, \mathrm{n}, \mathrm{N} \in \mathbb{R}$ with $\mathrm{mM}, \mathrm{nN}>0$. If $(\mathrm{A}, \mathrm{C})$ has the trace $\mathrm{P}-(\mathrm{m}, \mathrm{M})$-property and $(\mathrm{B}, \mathrm{C})$ has the trace $\mathrm{P}-(\mathrm{n}, \mathrm{N})$-property, then

$$
\left|\frac{\operatorname{tr}(\mathrm{PBA}) \operatorname{tr}\left(\mathrm{PC}^{2}\right)}{\operatorname{tr}(\mathrm{PCA}) \operatorname{tr}(\mathrm{PBC})}-1\right| \leq \frac{1}{4} \cdot \frac{(\mathrm{M}-\mathrm{m})(\mathrm{N}-\mathrm{n})}{\sqrt{\mathrm{mnMN}}} .
$$


If $\mathrm{A}$ has the trace $\mathrm{P}-(\mathrm{k}, \mathrm{K})$-property and $\mathrm{B}$ has the trace $\mathrm{P}-(\mathrm{l}, \mathrm{L})$-property, then

$$
\left|\frac{\operatorname{tr}(\mathrm{PBA}) \operatorname{tr}(\mathrm{P})}{\operatorname{tr}(\mathrm{PA}) \operatorname{tr}(\mathrm{PB})}-1\right| \leq \frac{1}{4} \cdot \frac{(\mathrm{K}-\mathrm{k})(\mathrm{L}-\mathrm{l})}{\sqrt{\mathrm{klKL}}},
$$

where $\mathrm{kK}, \mathrm{lL}>0$.

We observe that, if $0<\mathrm{k} 1_{\mathrm{H}} \leq \mathrm{A} \leq \mathrm{K} 1_{\mathrm{H}}$ and $0<\mathrm{l} 1_{\mathrm{H}} \leq \mathrm{B} \leq \mathrm{L} 1_{\mathrm{H}}$, then by (46) we have

$$
|\operatorname{tr}(\mathrm{PBA}) \operatorname{tr}(\mathrm{P})-\operatorname{tr}(\mathrm{PA}) \operatorname{tr}(\mathrm{PB})| \leq \frac{1}{4} \cdot \frac{(\mathrm{K}-\mathrm{k})(\mathrm{L}-\mathrm{l})}{\sqrt{\mathrm{klKL}}} \operatorname{tr}(\mathrm{PA}) \operatorname{tr}(\mathrm{PB})
$$

or, equivalently

$$
\left|\frac{\operatorname{tr}(\mathrm{PBA})}{\operatorname{tr}(\mathrm{P})}-\frac{\operatorname{tr}(\mathrm{PA})}{\operatorname{tr}(\mathrm{P})} \frac{\operatorname{tr}(\mathrm{PB})}{\operatorname{tr}(\mathrm{P})}\right| \leq \frac{1}{4} \cdot \frac{(\mathrm{K}-\mathrm{k})(\mathrm{L}-\mathrm{l})}{\sqrt{\mathrm{klKL}}} \frac{\operatorname{tr}(\mathrm{PA})}{\operatorname{tr}(\mathrm{P})} \frac{\operatorname{tr}(\mathrm{PB})}{\operatorname{tr}(\mathrm{P})} .
$$

\section{Applications for convex functions}

In the paper [30] we obtained amongst other the following reverse of the Jensen trace inequality:

$$
\begin{aligned}
& 0 \leq \frac{\operatorname{tr}(\operatorname{Pf}(A))}{\operatorname{tr}(P)}-f\left(\frac{\operatorname{tr}(P A)}{\operatorname{tr}(P)}\right) \\
& \leq \frac{\operatorname{tr}\left(\mathrm{Pf}^{\prime}(\mathrm{A}) \mathrm{A}\right)}{\operatorname{tr}(\mathrm{P})}-\frac{\operatorname{tr}(\mathrm{PA})}{\operatorname{tr}(\mathrm{P})} \cdot \frac{\operatorname{tr}\left(\mathrm{Pf}^{\prime}(\mathrm{A})\right)}{\operatorname{tr}(\mathrm{P})} \\
& \leq\left\{\begin{array}{l}
\frac{1}{2}\left[f^{\prime}(M)-f^{\prime}(m)\right] \frac{\operatorname{tr}\left(P\left|A-\frac{\operatorname{tr}(P A)}{\operatorname{tr}(P)} 1_{H}\right|\right)}{\operatorname{tr}(P)} \\
\frac{1}{2}(M-m) \frac{\operatorname{tr}\left(P\left|f^{\prime}(A)-\frac{\operatorname{tr}\left(P f^{\prime}(A)\right)}{\operatorname{tr}(P)} 1_{H}\right|\right)}{\operatorname{tr}(P)}
\end{array}\right. \\
& \leq\left\{\begin{array}{l}
\frac{1}{2}\left[f^{\prime}(M)-f^{\prime}(m)\right]\left[\frac{\operatorname{tr}\left(\mathrm{PA}^{2}\right)}{\operatorname{tr}(\mathrm{P})}-\left(\frac{\operatorname{tr}(\mathrm{PA})}{\operatorname{tr}(\mathrm{P})}\right)^{2}\right]^{1 / 2} \\
\frac{1}{2}(\mathrm{M}-\mathrm{m})\left[\frac{\operatorname{tr}\left(\mathrm{P}\left[\mathrm{f}^{\prime}(\mathrm{A})\right]^{2}\right)}{\operatorname{tr}(\mathrm{P})}-\left(\frac{\operatorname{tr}\left(\mathrm{Pf}^{\prime}(\mathrm{A})\right)}{\operatorname{tr}(\mathrm{P})}\right)^{2}\right]^{1 / 2}
\end{array}\right. \\
& \leq \frac{1}{4}\left[f^{\prime}(M)-f^{\prime}(m)\right](M-m) .
\end{aligned}
$$

Let $\mathcal{M}_{n}(\mathbb{C})$ be the space of all square matrices of order $n$ with complex elements and $A \in \mathcal{M}_{\mathrm{n}}(\mathbb{C})$ be a Hermitian matrix such that $\mathrm{Sp}(A) \subseteq[\mathrm{m}, \mathrm{M}]$ 
for some scalars $m, M$ with $m<M$. If $f$ is a continuously differentiable convex function on $[\mathrm{m}, M]$, then by taking $P=I_{n}$ in (48) we get

$$
\begin{aligned}
& 0 \leq \frac{\operatorname{tr}(f(A))}{n}-f\left(\frac{\operatorname{tr}(A)}{n}\right) \\
& \leq \frac{\operatorname{tr}\left(f^{\prime}(A) A\right)}{n}-\frac{\operatorname{tr}(A)}{n} \cdot \frac{\operatorname{tr}\left(f^{\prime}(A)\right)}{n} \\
& \leq\left\{\begin{array}{l}
\frac{1}{2}\left[f^{\prime}(M)-f^{\prime}(m)\right] \frac{\operatorname{tr}\left(\left|A-\frac{\operatorname{tr}(A)}{n} 1_{H}\right|\right)}{n} \\
\frac{1}{2}(M-m) \frac{\operatorname{tr}\left(\left|f^{\prime}(A)-\frac{\operatorname{tr}\left(f^{\prime}(A)\right)}{n} 1_{H}\right|\right)}{n}
\end{array}\right. \\
& \leq\left\{\begin{array}{l}
\frac{1}{2}\left[f^{\prime}(M)-f^{\prime}(m)\right]\left[\frac{\operatorname{tr}\left(A^{2}\right)}{n}-\left(\frac{\operatorname{tr}(A)}{n}\right)^{2}\right]^{1 / 2} \\
\frac{1}{2}(M-m)\left[\frac{\operatorname{tr}\left(\left[f^{\prime}(A)\right]^{2}\right)}{n}-\left(\frac{\operatorname{tr}\left(f^{\prime}(A)\right)}{n}\right)^{2}\right]^{1 / 2}
\end{array}\right. \\
& \leq \frac{1}{4}\left[f^{\prime}(M)-f^{\prime}(m)\right](M-m) \text {. }
\end{aligned}
$$

The following reverse inequality also holds:

Proposition 1 Let $\mathrm{A}$ be a selfadjoint operator on the Hilbert space $\mathrm{H}$ and assume that $\mathrm{Sp}(\mathrm{A}) \subseteq[\mathrm{m}, \mathrm{M}]$ for some scalars $\mathrm{m}, \mathrm{M}$ with $0<\mathrm{m}<\mathrm{M}$. If $\mathrm{f}$ is a continuously differentiable convex function on $[\mathrm{m}, \mathrm{M}]$ with $\mathrm{f}^{\prime}(\mathrm{m})>0$ and $\mathrm{P} \in \mathcal{B}_{1}(\mathrm{H}) \backslash\{0\}, \mathrm{P} \geq 0$, then we have

$$
\begin{aligned}
0 & \leq \frac{\operatorname{tr}(P f(A))}{\operatorname{tr}(P)}-f\left(\frac{\operatorname{tr}(P A)}{\operatorname{tr}(P)}\right) \\
& \leq \frac{\operatorname{tr}\left(P f^{\prime}(A) A\right)}{\operatorname{tr}(P)}-\frac{\operatorname{tr}(P A)}{\operatorname{tr}(P)} \cdot \frac{\operatorname{tr}\left(P f^{\prime}(A)\right)}{\operatorname{tr}(P)} \\
& \leq \frac{1}{4} \cdot \frac{(M-m)\left[f^{\prime}(M)-f^{\prime}(m)\right]}{\sqrt{m M f^{\prime}(m) f^{\prime}(M)}} \frac{\operatorname{tr}(P A)}{\operatorname{tr}(P)} \frac{\operatorname{tr}\left(P f^{\prime}(A)\right)}{\operatorname{tr}(P)} .
\end{aligned}
$$

The proof follows by the inequality (47) and the details are omitted.

Let $A \in \mathcal{M}_{\mathrm{n}}(\mathbb{C})$ be a Hermitian matrix such that $S p(A) \subseteq[m, M]$ for some scalars $m, M$ with $m<M$. If $f$ is a continuously differentiable convex function 
on $[\mathrm{m}, \mathrm{M}]$ with $\mathrm{f}^{\prime}(\mathrm{m})>0$ then by taking $\mathrm{P}=\mathrm{I}_{\mathrm{n}}$ in (50) we get

$$
\begin{aligned}
0 & \leq \frac{\operatorname{tr}(f(A))}{n}-f\left(\frac{\operatorname{tr}(A)}{n}\right) \\
& \leq \frac{\operatorname{tr}\left(f^{\prime}(A) A\right)}{n}-\frac{\operatorname{tr}(A)}{n} \cdot \frac{\operatorname{tr}\left(f^{\prime}(A)\right)}{n} \\
& \leq \frac{1}{4} \cdot \frac{(M-m)\left[f^{\prime}(M)-f^{\prime}(m)\right]}{\sqrt{m M f^{\prime}(m) f^{\prime}(M)}} \frac{\operatorname{tr}(A)}{n} \frac{\operatorname{tr}\left(f^{\prime}(A)\right)}{n} .
\end{aligned}
$$

We consider the power function $f:(0, \infty) \rightarrow(0, \infty), f(t)=t^{r}$ with $t \in$ $\mathbb{R} \backslash\{0\}$. For $r \in(-\infty, 0) \cup[1, \infty), f$ is convex while for $r \in(0,1), f$ is concave.

Let $r \geq 1$ and $A$ be a selfadjoint operator on the Hilbert space $H$ and assume that $S p(A) \subseteq[m, M]$ for some scalars $m, M$ with $0<m<M$. If $\mathrm{P} \in \mathcal{B}_{1}^{+}(\mathrm{H}) \backslash\{0\}$, then

$$
\begin{aligned}
0 & \leq \frac{\operatorname{tr}\left(\mathrm{PA}^{\mathrm{r}}\right)}{\operatorname{tr}(\mathrm{P})}-\left(\frac{\operatorname{tr}(\mathrm{PA})}{\operatorname{tr}(\mathrm{P})}\right)^{\mathrm{r}} \\
& \leq \mathrm{r}\left[\frac{\operatorname{tr}\left(\mathrm{PA}^{\mathrm{r}}\right)}{\operatorname{tr}(\mathrm{P})}-\frac{\operatorname{tr}(\mathrm{PA})}{\operatorname{tr}(\mathrm{P})} \cdot \frac{\operatorname{tr}\left(\mathrm{PA}^{\mathrm{r}-1}\right)}{\operatorname{tr}(\mathrm{P})}\right] \\
& \leq \frac{1}{4} \mathrm{r} \frac{(\mathrm{M}-\mathrm{m})\left(\mathrm{M}^{\mathrm{r}-1}-\mathrm{m}^{\mathrm{r}-1}\right)}{\mathrm{m}^{\mathrm{r} / 2} \mathrm{M}^{\mathrm{r} / 2}} \frac{\operatorname{tr}(\mathrm{PA})}{\operatorname{tr}(\mathrm{P})} \frac{\operatorname{tr}\left(\mathrm{PA}^{\mathrm{r}-1}\right)}{\operatorname{tr}(\mathrm{P})} .
\end{aligned}
$$

If we take the first and last term in (52) we get the inequality:

$$
\begin{aligned}
0 & \leq \frac{\operatorname{tr}(\mathrm{P}) \operatorname{tr}\left(\mathrm{PA}^{\mathrm{r}}\right)}{\operatorname{tr}(\mathrm{PA}) \operatorname{tr}\left(\mathrm{PA}^{\mathrm{r}-1}\right)}-\frac{\operatorname{tr}(\mathrm{P})[\operatorname{tr}(\mathrm{PA})]^{\mathrm{r}-1}}{\operatorname{tr}\left(\mathrm{PA}^{\mathrm{p}-1}\right)[\operatorname{tr}(\mathrm{P})]^{\mathrm{r}-1}} \\
& \leq \frac{1}{4} \mathrm{r} \frac{(\mathrm{M}-\mathrm{m})\left(\mathrm{M}^{\mathrm{r}-1}-\mathrm{m}^{\mathrm{r}-1}\right)}{\mathrm{m}^{\mathrm{r} / 2} \mathrm{M}^{\mathrm{r} / 2}} .
\end{aligned}
$$

\section{References}

[1] G. A. Anastassiou, Grüss type inequalities for the Stieltjes integral, Nonlinear Funct. Anal. Appl., 12 (2007), no. 4, 583-593.

[2] G. A. Anastassiou, Chebyshev-Grüss type and comparison of integral means inequalities for the Stieltjes integral, Panamer. Math. J., 17 (2007), no. 3, 91-109.

[3] G. A. Anastassiou, Chebyshev-Grüss type inequalities via Euler type and Fink identities, Math. Comput. Modelling, 45 (2007), no. 9-10, 1189-1200 
[4] T. Ando, Matrix Young inequalities, Oper. Theory Adv. Appl., 75 (1995), $33-38$.

[5] R. Bellman, Some inequalities for positive definite matrices, in: E.F. Beckenbach (Ed.), General Inequalities 2, Proceedings of the 2nd International Conference on General Inequalities, Birkhäuser, Basel, 1980, pp. 89-90.

[6] E. V. Belmega, M. Jungers, S. Lasaulce, A generalization of a trace inequality for positive definite matrices, Aust. J. Math. Anal. Appl., 7 (2010), no. 2, Art. 26, 5 pp.

[7] N. G. de Bruijn, Problem 12, Wisk. Opgaven, 21 (1960), 12-14.

[8] P. Cerone, S. S. Dragomir, A refinement of the Grüss inequality and applications. Tamkang J. Math., 38 (2007), no. 1, 37-49.

[9] P. Cerone, S. S. Dragomir, New bounds for the Čebyšev functional. Appl. Math. Lett., 18 (2005), no. 6, 603-611.

[10] P. Cerone, S. S. Dragomir, Chebychev functional bounds using Ostrowski seminorms, Southeast Asian Bull. Math., 28 (2004), no. 2, 219-228.

[11] D. Chang, A matrix trace inequality for products of Hermitian matrices, J. Math. Anal. Appl., 237 (1999) 721-725.

[12] L. Chen, C. Wong, Inequalities for singular values and traces, Linear Algebra Appl., 171 (1992), 109-120.

[13] I. D. Coop, On matrix trace inequalities and related topics for products of Hermitian matrix, J. Math. Anal. Appl., 188 (1994), 999-1001.

[14] S. S. Dragomir, Some Grüss type inequalities in inner product spaces, J. Inequal. Pure 83 Appl. Math., 4(2003), No. 2, Article 42, [ON LINE: http://jipam.vu.edu.au/v4n2/032_03.html].

[15] S. S. Dragomir, A Survey on Cauchy-Bunyakovsky-Schwarz Type Discrete Inequalities, RGMIA Monographs, Victoria University, 2002. (ON LINE: http://rgmia.vu.edu.au/monographs/) .

[16] S. S. Dragomir, A counterpart of Schwarz's inequality in inner product spaces, East Asian Math. J., 20 (1) (2004), 1-10. Zbl 1094.26017. Preprint RGMIA Res. Rep. Coll., 6(2003), Supplement, Article 18. [ON LINE: http://rgmia.vu.edu.au/v6(E).html] . 
[17] S. S. Dragomir, Grüss inequality in inner product spaces, The Australian Math Soc. Gazette, 26 (1999), No. 2, 66-70.

[18] S. S. Dragomir, A generalization of Grüss' inequality in inner product spaces and applications, J. Math. Anal. Appl., 237 (1999), 74-82.

[19] S. S. Dragomir, Some discrete inequalities of Grüss type and applications in guessing theory, Honam Math. J., 21 (1) (1999), 145-156.

[20] S. S. Dragomir, Some integral inequalities of Grüss type, Indian J. of Pure and Appl. Math., 31 (4) (2000), 397-415.

[21] S. S. Dragomir, Advances in Inequalities of the Schwarz, Grüss and Bessel Type in Inner Product Spaces, Nova Science Publishers Inc., New York, 2005, x+249 pp.

[22] S. S. Dragomir, G. L. Booth, On a Grüss-Lupaş type inequality and its applications for the estimation of p-moments of guessing mappings, Mathematical Communications, 5 (2000), 117-126.

[23] S. S. Dragomir, A Grüss type integral inequality for mappings of $r$ Hölder's type and applications for trapezoid formula, Tamkang J. of Math., 31 (1) (2000), 43-47.

[24] S. S. Dragomir, I. Fedotov, An inequality of Grüss' type for RiemannStieltjes integral and applications for special means, Tamkang J. of Math., 29 (4) (1998), 286-292.

[25] S. S. Dragomir, Čebyšev's type inequalities for functions of selfadjoint operators in Hilbert spaces, Linear Multilinear Algebra, 58 (2010), no. 7-8, 805-814. Preprint RGMIA Res. Rep. Coll., 11 (2008), Supp. Art. 9. [Online http://rgmia.org/v11(E).php]

[26] S. S. Dragomir, Grüss' type inequalities for functions of selfadjoint operators in Hilbert spaces, Ital. J. Pure Appl. Math. No. 28 (2011), 207224. Preprint RGMIA Res. Rep. Coll. 11 (2008), Supp. Art. 11. [Online http://rgmia.org/v11(E).php]

[27] S. S. Dragomir, New inequalities of the Kantorovich type for bounded linear operators in Hilbert spaces, Linear Algebra Appl. 428 (2008), no. 11-12, 2750-2760. 
[28] S. S. Dragomir, Some Čebyšev's type trace inequalities for functions of selfadjoint operators in Hilbert spaces, Preprint RGMIA Res. Rep. Coll. 17 (2014), Art. 111. [Online http://rgmia.org/papers/v17/v17a111.pdf] .

[29] S. S. Dragomir, Some Grüss type inequalities for trace of operators in Hilbert spaces, Preprint RGMIA Res. Rep. Coll. 17 (2014), Art. 114. [Online http://rgmia.org/papers/v17/v17a114.pdf] .

[30] S. S. Dragomir, Reverse Jensen's type trace inequalities for convex functions of selfadjoint operators in Hilbert spaces, Preprint RGMIA Res. Rep. Coll. 17 (2014), Art. 118. [Online http://rgmia.org/papers/v17/v17a118.pdf] .

[31] A. M. Fink, A treatise on Grüss' inequality, Analytic and Geometric Inequalities, 93-113, Math. Appl. 478, Kluwer Academic Publ., 1999.

[32] S. Furuichi, M. Lin, Refinements of the trace inequality of Belmega, Lasaulce and Debbah. Aust. J. Math. Anal. Appl., 7 (2010), no. 2, Art. 23, 4 pp.

[33] T. Furuta, J. Mićić Hot, J. Pečarić, Y. Seo, Mond-Pečarić Method in Operator Inequalities. Inequalities for Bounded Selfadjoint Operators on a Hilbert Space, Element, Zagreb, 2005.

[34] W. Greub and W. Rheinboldt, On a generalisation of an inequality of L.V. Kantorovich, Proc. Amer. Math. Soc., 10 (1959), 407-415.

[35] G. Grüss, Über das Maximum des absoluten Betrages von $\frac{1}{b-a} \int_{a}^{b} f(x) g(x) d x-\frac{1}{(b-a)^{2}} \int_{a}^{b} f(x) d x \int_{a}^{b} g(x) d x$, Math. Z., 39 (1935), 215-226.

[36] H. D. Lee, On some matrix inequalities, Korean J. Math., 16 (2008), No. $4,565-571$.

[37] L. Liu, A trace class operator inequality, J. Math. Anal. Appl., 328 (2007), 1484-1486.

[38] Z. Liu, Refinement of an inequality of Grüss type for Riemann-Stieltjes integral, Soochow J. Math., 30 (4) (2004), 483-489.

[39] S. Manjegani, Hölder and Young inequalities for the trace of operators, Positivity, 11 (2007), 239-250. 
[40] A. Matković, J. Pečarić, I. Perić, A variant of Jensen's inequality of Mercer's type for operators with applications, Linear Algebra Appl., 418 (2006), no. 2-3, 551-564.

[41] D. S. Mitrinović, J. E. Pečarić, A.M. Fink, Classical and New Inequalities in Analysis, Kluwer Academic Publishers, Dordrecht, 1993.

[42] H. Neudecker, A matrix trace inequality, J. Math. Anal. Appl., 166 (1992), 302-303.

[43] J. Pečarić, J. Mićić, Y. Seo, Inequalities between operator means based on the Mond-Pečarić method. Houston J. Math., 30 (2004), no. 1, 191-207

[44] G. Pólya, G. Szegö, Aufgaben und Lehrsätze aus der Analysis, Vol. 1, Berlin 1925, pp. 57 and 213-214.

[45] M. B. Ruskai, Inequalities for traces on von Neumann algebras, Commun. Math. Phys. 26 (1972), 280-289.

[46] K. Shebrawi, H. Albadawi, Operator norm inequalities of Minkowski type, J. Inequal. Pure Appl. Math., 9 (1) (2008), 1-10, article 26.

[47] K. Shebrawi, H. Albadawi, Trace inequalities for matrices, Bull. Aust. Math. Soc., 87 (2013), 139-148.

[48] O. Shisha and B. Mond, Bounds on differences of means, Inequalities I, New York-London, 1967, 293-308.

[49] B. Simon, Trace Ideals and Their Applications, Cambridge University Press, Cambridge, 1979.

[50] Z. Ulukök and R. Türkmen, On some matrix trace inequalities. J. Inequal. Appl., 2010, Art. ID 201486, 8 pp.

[51] X. Yang, A matrix trace inequality, J. Math. Anal. Appl., 250 (2000) $372-374$.

[52] X. M. Yang, X. Q. Yang, K. L. Teo, A matrix trace inequality, J. Math. Anal. Appl., 263 (2001), 327-331.

[53] Y. Yang, A matrix trace inequality, J. Math. Anal. Appl., 133 (1988) $573-574$. 